LTH-336

NUB-3094-94TH

KEK-TH-404

hep-ph/9407291

July 1994

\title{
Decoupling of the $\epsilon$-scalar mass in softly broken supersymmetry
}

\author{
Ian Jack, D. R. Timothy Jones, \\ DAMTP, University of Liverpool, Liverpool L69 3BX, U.K. \\ Stephen P. Martin, Michael T. Vaughn \\ Department of Physics, Northeastern University, Boston, MA 02115, USA \\ and \\ Youichi Yamada周 \\ Theory Group, KEK, Tsukuba, Ibaraki 305, Japan
}

\begin{abstract}
It has been shown recently that the introduction of an unphysical $\epsilon$-scalar mass $\tilde{m}$ is necessary for the proper renormalization of softly broken supersymmetric theories by dimensional reduction $(\overline{\mathrm{DR}})$. In these theories, both the two-loop $\beta$-functions of the scalar masses and their one-loop finite corrections depend on $\tilde{m}^{2}$. We find, however, that the dependence on $\tilde{m}^{2}$ can be completely removed by slightly modifying the $\overline{\mathrm{DR}}$ renormalization scheme. We also show that previous $\overline{\mathrm{DR}}$ calculations of one-loop corrections in supersymmetry which ignored the $\tilde{m}^{2}$ contribution correspond to using this modified scheme.
\end{abstract}

*Fellow of the Japan Society for the Promotion of Science 
The usual regularization procedure for the renormalization of softly broken supersymmetric theories is dimensional reduction [1] since it respects supersymmetry (SUSY), modulo possible high-loop ambiguities which will not concern us here[2]. In this regularization, an originally 4-dimensional vector field is split into two fields: the first $D=4-2 \epsilon$ components (a $D$-dimensional vector) and the last $2 \epsilon$ components ( $\epsilon$-scalars). All momentum integrals are $D$-dimensional. The $\epsilon$-scalars are differently renormalized in non-supersymmetric theories, and their interactions, called evanescent couplings, should in general be treated as independent of the corresponding interactions of the vector fields.

In the renormalization of softly broken supersymmetric theories, all the dimensionless evanescent couplings are related to the vector couplings by SUSY and are therefore not independent. For the soft SUSY breaking terms, however, there is one possible independent evanescent coupling, the mass $\tilde{m}$ of the $\epsilon$-scalars. 17 Moreover, we cannot set $\tilde{m}=0$ in the renormalization group analysis [3], and the two-loop $\beta$ function of scalar masses in the $\overline{\mathrm{DR}}$ scheme (dimensional reduction with modified minimal subtraction (4⿴囗十) depends explicitly on $\tilde{m}^{2}$. In this paper, however, we will show that the $\tilde{m}^{2}$ dependence can be removed from both the two-loop $\beta$ functions of the physical couplings and the corresponding one-loop finite corrections by a slight modification of the renormalization scheme. Therefore in this modified scheme we need not consider the explicit value of $\tilde{m}^{2}$.

We first present the problem of the $\epsilon$-scalar masses. Let us consider a supersymmetric theory with the gauge group a product of simple [or $U(1)]$ factors $G_{A}$, each with a vector supermultiplet $\left(V_{\mu}^{A a}, \chi^{A a}\right)$ (the index $a$ runs over the adjoint representation of $\left.G_{A}\right)$. There are also chiral supermultiplets $\Phi_{i}=\left(\phi_{i}, \psi_{i}\right)$ in representations (reducible, in general) of $G_{A}$ with generators $\left(R^{A a}\right)_{i}^{j}$. The superpotential $W$ and the

\footnotetext{
${ }^{\dagger}$ If there is a chiral multiplet which transforms according to a representation of the gauge group contained in the symmetric product of two adjoints, then there is also a cubic interaction which must be considered [3]. This possibility does not arise in the supersymmetric standard model, however, so we ignore it here for simplicity.
} 
soft supersymmetric breaking terms $\mathcal{L}_{\text {soft }}$ are

$$
W=\frac{1}{6} \lambda^{i j k} \Phi_{i} \Phi_{j} \Phi_{k}+\frac{1}{2} \mu^{i j} \Phi_{i} \Phi_{j}
$$

and

$$
\mathcal{L}_{\text {soft }}=-\left(m^{2}\right)_{i}^{j} \phi^{* i} \phi_{j}-\left(\frac{1}{6} h^{i j k} \phi_{i} \phi_{j} \phi_{k}+\frac{1}{2} b^{i j} \phi_{i} \phi_{j}+\frac{1}{2} M_{A} \chi^{A a} \chi^{A a}+\text { h.c. }\right)
$$

respectively. (We assume there are no gauge singlet chiral supermultiplets so there are no terms linear in $\phi$.) As shown in Ref. [3], use of dimensional reduction means that we have to add an independent evanescent coupling

$$
\mathcal{L}_{\epsilon}=\frac{1}{2} \tilde{m}_{A}^{2} V_{\sigma}^{A a} V^{A a \sigma}
$$

the mass term for the $\epsilon$-scalars, to the usual $\mathcal{L}_{\text {soft }}$. Here the index $\sigma$ runs over the last $2 \epsilon$ components of the original 4-dimensional vector index.

The $\epsilon$-scalar mass term gives contributions to the next-to-leading order corrections to the scalar masses. Firstly, the two-loop $\beta$ function for $m^{2}$ is given by [5, 6, 3]

$$
\begin{aligned}
(4 \pi)^{4} \beta \frac{(2)}{\mathrm{DR}}\left(m^{2}\right)_{i}^{j}= & 16 \delta_{i}^{j} g_{A}^{2} g_{B}^{2} C_{A}(i) C_{B}(i)\left(2\left|M_{A}\right|^{2}+M_{A} M_{B}^{*}\right) \\
& +24 \delta_{i}^{j} g_{A}^{4} C_{A}(i)\left[T_{A}(\Phi)-3 C_{A}(V)\right]\left|M_{A}\right|^{2} \\
& +g_{A}^{2}\left[2 C_{A}(l)-C_{A}(i)\right]\left[\lambda_{i k l}^{*} \lambda^{i^{\prime} k l}\left(m^{2}\right)_{i^{\prime}}^{j}+\lambda_{j^{\prime} k l}^{*} \lambda^{j k l}\left(m^{2}\right)_{i}^{j^{\prime}}\right] \\
& -\frac{1}{2}\left[\lambda_{i k l}^{*} \lambda^{l s t} \lambda_{q s t}^{*} \lambda^{i^{\prime} k q}\left(m^{2}\right)_{i^{\prime}}^{j}+\lambda_{j^{\prime} k l}^{*} \lambda^{l s t} \lambda_{q s t}^{*} \lambda^{j k q}\left(m^{2}\right)_{i}^{j^{\prime}}\right] \\
& +2 g_{A}^{2}\left[2 C_{A}(l)-C_{A}(i)\right]\left[2 \lambda_{i k l}^{*} \lambda^{j k l}\left|M_{A}\right|^{2}-h_{i k l}^{*} \lambda^{j k l} M_{A}\right. \\
& \left.-\lambda_{i k l}^{*} h^{j k l} M_{A}^{*}+h_{i k l}^{*} h^{j k l}+\lambda_{i k l}^{*}\left(m^{2}\right)_{k^{\prime}}^{k} \lambda^{j k^{\prime} l}+\lambda_{i k l}^{*}\left(m^{2}\right)_{l^{\prime}}^{l} \lambda^{j k l^{\prime}}\right] \\
& -h_{i k l}^{*} h^{l s t} \lambda_{q s t}^{*} \lambda^{j k q}-h_{i k l}^{*} \lambda^{l s t} \lambda_{q s t}^{*} h^{j k q}-\lambda_{i k l}^{*} h^{l s t} h_{q s t}^{*} \lambda^{j k q} \\
& -\lambda_{i k l}^{*} \lambda^{l s t} h_{q s t}^{*} h^{j k q}-\lambda_{i k l}^{*}\left(m^{2}\right)_{k^{\prime}}^{k} \lambda^{l s t} \lambda_{q s t}^{*} \lambda^{j k^{\prime} q}-\lambda_{i k l}^{*}\left(m^{2}\right)_{l^{\prime}}^{l} \lambda^{l^{\prime} s t} \lambda_{q s t}^{*} \lambda^{j k q} \\
& -\lambda_{i k l}^{*} \lambda^{l s t} \lambda_{q s t}^{*}\left(m^{2}\right)_{q^{\prime}}^{q} \lambda^{j k q^{\prime}}-2 \lambda_{i k l}^{*} \lambda^{l s t}\left(m^{2}\right)_{t}^{t^{\prime}} \lambda_{q s t^{\prime}}^{*} \lambda^{j k q} \\
& -2 g_{A}^{2}\left(R^{A a}\right)_{i}^{j}\left(R^{A a} m^{2}\right)_{r}^{l} \lambda_{l p q}^{*} \lambda^{r p q}+8 g_{A}^{2} g_{B}^{2}\left(R^{A a}\right)_{i}^{j} \operatorname{Tr}\left[R^{A a} C_{B}(r) m^{2}\right]
\end{aligned}
$$




$$
\begin{aligned}
& +16 \delta_{i}^{j} g_{A}^{4} C_{A}(i)\left(\operatorname{Tr}\left[C_{A}(r) m^{2}\right] / d\left(G_{A}\right)-C_{A}(V)\left|M_{A}\right|^{2}\right) \\
& +8 \delta_{i}^{j} g_{A}^{4} C_{A}(i)\left[T_{A}(\Phi)-3 C_{A}(V)\right] \tilde{m}_{A}^{2} \\
& -2 g_{A}^{2}\left[C_{A}(i)+2 C_{A}(l)\right] \lambda_{i k l}^{*} \lambda^{j k l} \tilde{m}_{A}^{2} .
\end{aligned}
$$

Here the $C_{A}(i)$ are the eigenvalues of the Casimir operator defined by

$$
C_{A}(i) \delta_{i}^{j}=\left(R^{A a} R^{A a}\right)_{i}^{j}
$$

$C_{A}(V)$ is the eigenvalue on the adjoint representation of $G_{A}$, and $C_{A}(r)$ which appears in the trace terms denotes the eigenvalue of $C_{A}$ on the irreducible representations $r$ appearing in the trace. $T_{A}(\Phi)$ is the Dynkin index

$$
T_{A}(\Phi) \delta^{a b}=\operatorname{Tr}\left(R^{A a} R^{A b}\right)
$$

and $d\left(G_{A}\right)$ is the dimension of $G_{A}$.

In fact the proper inclusion of the $\epsilon$-scalar mass and its counterterms resolves the discrepancy between the result for $\beta^{(2)}\left(m^{2}\right)$ in [3] and those in the original versions of [5, 6].

Secondly, the one-loop pole masses of scalars also depend on $\tilde{m}^{2}$. The general expression is:

$$
m_{i}^{2}(\text { pole })=\left.m_{i}^{2}\right|_{\overline{\mathrm{DR}}}-\frac{2 g_{A}^{2} C_{A}(i)}{(4 \pi)^{2}} \tilde{m}_{A}^{2}-\Pi_{i}\left(q^{2}=m_{i}^{2}(\text { pole }), m_{i}^{2}\right) .
$$

Here $\Pi_{i}\left(q^{2}, m_{i}^{2}\right)$ is the one-loop two-point function of $\phi_{i}$ calculated in $\overline{\mathrm{DR}}$ with $\tilde{m}=0$. Assuming there are no gauge singlets, there are no other physical $\beta$ functions or finite corrections which depend on $\tilde{m}^{2}$.

Since the $\epsilon$-scalar mass is not an observable, the results (4) and (7) appear at first sight somewhat paradoxical. This issue was addressed in Ref.[7], where it was proved that $\overline{\mathrm{DR}}$ is related to the standard dimensional regularisation scheme $(\overline{\mathrm{MS}})[8$ by coupling constant redefinition. It follows that the S-matrix is identical in the two schemes. Nevertheless, the $\tilde{m}^{2}$ dependence is an inconvenience for the $\overline{\mathrm{DR}}$ scheme. 
Although it is safe simply to impose $\tilde{m}=0$ for calculations at a fixed renormalization scale, this condition is unstable under the renormalization group and may not be used in the renormalization group analyses. This is obvious from the one-loop $\beta$ function of $\tilde{m}^{2}$ [3],

$$
(4 \pi)^{2} \beta_{\overline{\mathrm{DR}}}^{(1)}\left(\tilde{m}_{A}^{2}\right)=2 g_{A}^{2}\left[T_{A}(\Phi)-3 C_{A}(V)\right] \tilde{m}_{A}^{2}+4 g_{A}^{2}\left(\operatorname{Tr}\left[C_{A}(r) m^{2}\right] / d\left(G_{A}\right)-C_{A}(V)\left|M_{A}\right|^{2}\right),
$$

which is inhomogeneous with respect to $\tilde{m}^{2}$.

One could of course use the $\overline{\mathrm{MS}}$ scheme, which is manifestly $\tilde{m}^{2}$-independent, but $\overline{\mathrm{MS}}$ does not respect SUSY and so a much more complicated treatment of the physical couplings would be entailed. What we would like is the best of both worlds: a prescription which respects SUSY and yet contains no $\tilde{m}^{2}$ dependence. One might expect that there is such a prescription, since $\tilde{m}^{2}$ is an unphysical artifact produced by dimensional reduction. We find that this is indeed the case. The $\tilde{m}^{2}$ dependences of (4) and (7) are removed by a simple modification of the renormalization scheme from the $\overline{\mathrm{DR}}$ scheme. Let us consider a new scheme $\left(\overline{\mathrm{DR}}^{\prime}\right)$ defined as

$$
\left.\left(m^{2}\right)_{i}^{j}\right|_{\overline{\mathrm{DR}}^{\prime}}=\left.\left(m^{2}\right)_{i}^{j}\right|_{\overline{\mathrm{DR}}}-\frac{2 g_{A}^{2} C_{A}(i)}{(4 \pi)^{2}} \delta_{i}^{j} \tilde{m}_{A}^{2}
$$

while all other couplings are not modified from $\overline{\mathrm{DR}}$. In fact, $\left.m^{2}\right|_{\overline{\mathrm{DR}}}$ is the same as the corresponding object in the dimensional regularization scheme $(\overline{\mathrm{MS}})$. The reason for this becomes clear with the realisation that we could arrive at $\overline{\mathrm{DR}}^{\prime}$ starting from $\overline{\mathrm{MS}}$, by making the redefinitions necessary to effect the change from $\overline{\mathrm{MS}}$ to $\overline{\mathrm{DR}}$ for all couplings except the scalar mass $\left(m^{2}\right)_{i}^{j}$. These redefinitions were given in [9]. Since $\overline{\mathrm{MS}}$ is manifestly $\tilde{m}^{2}$-independent, it follows that this scheme $\left(\overline{\mathrm{DR}}^{\prime}\right)$ will be too.

In the $\overline{\mathrm{DR}}^{\prime}$ scheme, the two-loop $\beta$ function for $m^{2}$ becomes

$$
\begin{aligned}
(4 \pi)^{4} \beta_{\overline{\mathrm{DR}}^{\prime}}^{(2)}\left(m^{2}\right)_{i}^{j}= & \left.(4 \pi)^{4} \beta_{\overline{\mathrm{DR}}}^{(2)}\left(m^{2}\right)_{i}^{j}\right) \\
& -8 \delta_{i}^{j} g_{A}^{4} C_{A}(i)\left(\operatorname{Tr}\left[C_{A}(r) m^{2}\right] / d\left(G_{A}\right)-C_{A}(V)\left|M_{A}\right|^{2}\right) \\
& -8 \delta_{i}^{j} g_{A}^{4} C_{A}(i)\left[T_{A}(\Phi)-3 C_{A}(V)\right] \tilde{m}_{A}^{2} \\
& +2 g_{A}^{2}\left[C_{A}(i)+2 C_{A}(l)\right] \lambda_{i k l}^{*} \lambda^{j k l} \tilde{m}_{A}^{2} .
\end{aligned}
$$


This $\beta_{\overline{\mathrm{DR}}^{\prime}}^{(2)}\left(m^{2}\right)$, which has no $\tilde{m}^{2}$ dependence, agrees with the result of Ref. [5]. Eq. (10) follows from (8), (9), and the one-loop $\beta$ function 10

$$
\begin{aligned}
(4 \pi)^{2} \beta^{(1)}\left(m^{2}\right)_{i}^{j}= & \frac{1}{2}\left[\lambda_{i k l}^{*} \lambda^{i^{\prime} k l}\left(m^{2}\right)_{i^{\prime}}^{j}+\lambda_{j^{\prime} k l}^{*} \lambda^{j k l}\left(m^{2}\right)_{i}^{j^{\prime}}\right]+2 \lambda_{i k l}^{*}\left(m^{2}\right)_{l^{\prime}}^{l} \lambda^{j k l^{\prime}} \\
& +h_{i k l}^{*} h^{j k l}-8 g_{A}^{2} C_{A}(i)\left|M_{A}\right|^{2} \delta_{i}^{j}+2 g_{A}^{2}\left(R^{A a}\right)_{i}^{j} \operatorname{Tr}\left(R^{A a} m^{2}\right) .
\end{aligned}
$$

The one-loop pole masses of scalars, given by

$$
m_{i}^{2}(\text { pole })=\left.m_{i}^{2}\right|_{\overline{\mathrm{DR}}^{\prime}}-\Pi_{i}\left(q^{2}=m_{i}^{2}(\text { pole }), m_{i}^{2}\right)
$$

are also $\tilde{m}^{2}$ independent.

The sets of $\left(m^{2}, \tilde{m}^{2}\right) \frac{}{\mathrm{DR}}$ which give the same $\left.m^{2}\right|_{\overline{\mathrm{DR}}^{\prime}}$ are of course physically equivalent. By comparing (12) with (7), we can also see that all the previous calculations of one-loop mass corrections in SUSY, which have ignored the contribution of $\epsilon$-scalar mass, are then justified as calculations in this $\overline{\mathrm{DR}}^{\prime}$ scheme.

In summary, we have found that the $\epsilon$-scalar mass dependence of the two-loop $\beta$ functions and of one-loop finite corrections can be completely removed by a slight modification of the renormalization scheme from the $\overline{\mathrm{DR}}$ scheme. We have also shown that the previous calculations of the one-loop mass corrections which have ignored the $\epsilon$-scalar mass contribution are justified as being (unwittingly) calculations in this new renormalization scheme, $\overline{\mathrm{DR}}^{\prime}$.

\section{Acknowledgements}

Y. Y. would like to thank K. Hagiwara for fruitful discussions. The work of D. I. J. and D. R. T. J. is supported in part by PPARC. The work of S. P. M. and M. T. V. is supported in part by the National Science Foundation grants PHY-90-01439 and PHY-93-06906 and U. S. Department of Energy grant DE-FG02-85ER40233. The work of Y. Y. is supported in part by the Japan Society for the Promotion of Science and the Grant-in-Aid for Scientific Research from the Ministry of Education, Science and Culture of Japan No. 06-1923. 


\section{References}

[1] W. Siegel, Phys. Lett. 84B, 193 (1979);

D. M. Capper, D. R. T. Jones and P. van Nieuwenhuizen, Nucl. Phys. B167, $479(1980)$.

[2] W. Siegel, Phys. Lett. 94B, 37 (1980);

L. V. Avdeev, G. A. Chochia and A. A. Vladimirov, Phys. Lett. 105B, 272 (1981) .

[3] I. Jack and D. R. T. Jones, Liverpool preprint LTH 334 (1994) (to be published in Phys. Lett. B).

[4] W. A. Bardeen, A. J. Buras, D. W. Duke and T. Muta, Phys. Rev. D18, 3998 (1978) .

[5] S. P. Martin and M. T. Vaughn, Northeastern preprint NUB-3081-93TH (1993) (to be published in Phys. Rev. D).

[6] Y. Yamada, KEK preprint KEK-TH-383 (1994) (to be published in Phys. Rev. D).

[7] I. Jack, D. R. T. Jones and K. L. Roberts, Liverpool preprint LTH 329 (1994) (to be published in Z. Phys. C).

[8] G. 't Hooft and M. Veltman, Nucl. Phys. B44, 189 (1972) .

[9] S. P. Martin and M. T. Vaughn, Phys. Lett. 318B, 331 (1993) .

[10] K. Inoue, A. Kakuto, H. Komatsu and S. Takeshita, Prog. Theor. Phys. 68, 927 (1982) ; ibid. 70, 330 (1983(E)) ; ibid. 71, 413 (1984) . 